\title{
Decision support systems in clinical practice: The case of venous thromboembolism prevention
}

\author{
G.I. Nazarenko, , E.B. Kleymenova ${ }^{a}$, S.A. Payushik ${ }^{a}$, V.A. Otdelenov ${ }^{a}$, D.A. Sychev ${ }^{b}$ \\ and L.P. Yashina ${ }^{\mathrm{c}, *}$ \\ ${ }^{\mathrm{a}}$ Medical Center of the Bank of Russia, Moscow, Russia \\ ${ }^{\mathrm{b}}$ Russian Medical Academy of Postgraduate Education, Moscow, Russia \\ 'Institute for Systems Analysis RAS, Moscow, Russia \\ *Corresponding author. E-mail: lyashina1@yandex.ru
}

BACKGROUND: Today medicine is facing a "knowledge crisis" in that explosively expanding medical knowledge encounters limited abilities to disseminate new practices [1]. Clinical practice guidelines (CPGs) are intended to promote high standards of care in specific areas of medicine by summarizing best clinical practice based on careful reviews of current research. However, doctors are often short of time to study these documents and check their updates, have little motivation for strict adherence to them. A systematic review of 11 studies reporting on 29 recommendations has found that median adherence to all recommendations was $34 \%$, suggesting that potential benefits for patients from health research may be lost [2].

Clinical decision support systems (CDSS) can serve as a knowledge translation tool, mediator between clinical guidelines and physicians by providing the right information to the right person at the right time.

OBJECTIVE: To evaluate the effectiveness of implementation of international and national CPGs for venous thromboembolism (VTE) prevention with the help of CDSS in a general hospital.

METHODS: A multifunctional CDSS based on national and international guidelines on the VTE prevention was developed and implemented in the Medical Center of the Bank of Russia (MC). The system has the following functionalities: 1) it supports the decision on the VTE prevention based on individual risk assessment of thrombosis (scales of Caprini, Rogers and Khorana, Padua Prediction Score, additional risk factors) and bleeding (IMPROVE scale for non-surgical patients, major bleeding scale for surgical patients and major orthopedic surgeries, hemorrhagic complications risk in cancer patients); 2) generates the summary containing the grade of recommendations and the level of evidence, personalized recommendations on regimen and duration of preventive antithrombotic therapy, dose correction according to creatinine clearance; 3 ) provides an audit form for and statistical analysis of VTE cases; 3) automatically generates a quality register for VTE prevention.

CDSS was implemented in June 2014. We analyzed VTE cases identified by triggers (deep vein thrombosis diagnosed by Doppler ultrasound and pulmonary embolism at the chest CT) that occurred in 2014 before and after CDSS implementation, as well as in the first half of 2015. Patients with VTE diagnosed during the first 48 hours of hospitalization or receiving anticoagulants in therapeutic doses were excluded from the analysis. Chi-square test for linear trend and non-parametric methods of descriptive statistics were used for data analysis. 
RESULTS: CDSS utilization was regulated by a special hospital-wide policy; lectures were organized to educate doctors how to use the system. Although international recommendations require VTE risk assessment for all hospitalized patients (except those receiving anticoagulant in therapeutic doses), the doctors filled forms for only 306 patients during the first 6 months of CDSS functioning $(14.1 \%$ of discharges with length of stay $>48$ hours during this period). In the first half of 2015 the coverage of VTE risk assessment with CDSS was 19\% $(n=506)$. Correctness of filling out the forms was $78.4 \%$, in the rest of cases doctors made mistakes in choosing patient's profile or when filling in risk scales.

Doctors adhere to given recommendations in $85.4 \%$ of cases. Most often (47.5\%) pharmacotherapy with low molecular weight heparin (LMWH), preventive doses, was recommended by the system, and in this category the adherence to recommended practice was the lowest $(74.6 \%)$. Among patients who underwent pharmacoprophylaxis, in $21.1 \%$ cases the use of anticoagulants was inconsistent with clinical guidelines or drug package insert (typically inappropriate choice of LMWH prophylactic doses, delaying or reducing the duration of prophylaxis).

The rate of hospital-acquired VTE significantly decreased after CDSS implementation and was 11.71, 8.28 and 4.84 per 1,000 hospitalizations in the first and second half of 2014 and in the first half of 2015, respectively $\left(\chi^{2}=7.325, \mathrm{df}=1, p=0.0068\right)$. The rate of postoperative VTE for the same period amounted to $8.76,3.39$ and 4.17 per 1,000 operations, respectively $\left(\chi^{2}=7.266, \mathrm{df}=1, p=0.007\right)$, reaching a level of the correspondent AHRQ safety indicator (4.99 per 1,000 operations) [3]. Deviations from clinical guidelines or anticoagulant package inserts were revealed in $74 \%$ of VTE cases; and more than 1/3 of deviations affected treatment outcomes.

CONCLUSIONS: Coverage of hospitalized patients with documented VTE risk assessment gradually increased after the CDSS implementation, but remained at a low level (19\% of eligible patients). Partly it may be attributed to the lack of CDSS integration in electronic health record or computerized physician order entry systems that would facilitate routine documentation of VTE and bleeding risks. However, the introduction of CDSS has allowed reducing significantly the rate of hospital-acquired VTE. This can be explained by drawing doctor's attention to the VTE problem and by training effect of CDSS. After receiving appropriate recommendations doctors adhere to them, on average, in $85.4 \%$ of cases, although for LMWH pharmacoprophylaxis this level was lower (74.6\%). Development of hospital-acquired VTE in most cases $(74 \%)$ was accompanied by non-compliance with CPGs recommendations, emphasizing the importance of additional measures for better adherence to evidence-based clinical practices.

Keywords: Decision support system, clinical practice guidelines, hospital-acquired, venous thromboembolism, prevention

Conflict of interest statement: The study was supported by the Ministry of Education and Science of the Russian Federation (project RFMEFI60714X0089) and by the RAS program of fundamental research "Bioinformatics, modern information technologies and mathematical methods in medicine".

\section{References}

[1] Fox J, Gutenstein M, Khan O, South M, Thomson R. OpenClinical.net: A platform for creating and sharing knowledge and promoting best practice in healthcare. Computers in Industry. 2015; 66: 63-72. doi:10.1016/j.compind.2014.10.001

[2] Mickan S, Burls A, Glasziou P. Patterns of 'leakage' in the utilisation of clinical guidelines: A systematic review. Postgrad Med J. 2011; 87: 670-9. doi: 10.1136/pgmj

[3] Agency for Healthcare Research and Quality. Patient safety indicators ${ }^{\mathrm{TM}}$ V5.0 benchmark data tables. Truven Health Analytics, 2015. 12 p. Available from: http://www.qualityindicators.ahrq.gov/Downloads/Modules/PSI/V50/Version_50_ Benchmark_Tables_PSI.pdf 